\title{
Adaptive motor cortex plasticity following grip reconstruction in individuals with tetraplegia
}

\author{
Lina Bunketorp Käll ${ }^{\mathrm{a}, \mathrm{b}, \mathrm{c}, *}$, Robert J. Cooper ${ }^{\mathrm{d}}$, Johanna Wangdell ${ }^{\mathrm{a}, \mathrm{e}}$, Jan Fridén ${ }^{\mathrm{a}, \mathrm{e}, \mathrm{f}}$ \\ and Malin Björnsdotter ${ }^{\mathrm{b}}$ \\ ${ }^{\mathrm{a}}$ Centre for Advanced Reconstruction of Extremities (C.A.R.E.), Sahlgrenska University Hospital/Mölndal, \\ Sweden \\ ${ }^{\mathrm{b}}$ Institute of Neuroscience and Physiology, Sahlgrenska Academy, University of Gothenburg, Gothenburg, Sweden \\ ${ }^{\mathrm{c}}$ MedTech West, Sahlgrenska University Hospital, Gothenburg, Sweden \\ ${ }^{\mathrm{d}}$ Department of Medical Physics and Biomedical Engineering, Biomedical Optics Research Laboratory, \\ University College London, London, United Kingdom \\ ${ }^{\mathrm{e}}$ Department of Hand Surgery, Institute of Clinical Sciences, Sahlgrenska Academy, University of Gothenburg, \\ Gothenburg, Sweden \\ ${ }^{\mathrm{f}}$ Swiss Paraplegic Centre, Nottwil, Switzerland
}

\begin{abstract}
.
Background: Tendon transfer is a surgical technique for restoring upper limb motor control in patients with cervical spinal cord injuries (SCI), and offers a rare window into cortical neuroplasticity following regained arm and hand function.

Objective: Here, we aimed to examine neuroplasticity mechanisms related to re-established voluntary motor control of thumb flexion following tendon transfer.

Methods: We used functional Magnetic Resonance Imaging (fMRI) to test the hypothesis that restored limb control following tendon transfer is mediated by activation of that limb's area of the primary motor cortex. We examined six individuals with tetraplegia who underwent right-sided surgical grip reconstruction at Sahlgrenska University Hospital, Sweden. All were right-handed males, with a SCI at the $\mathrm{C} 6$ or $\mathrm{C} 7$ level, and a mean age of 40 years (range $=31-48$ ). The average number of years elapsed since the SCI was 13 (range $=6-26$ ). Six right-handed gender- and age-matched control subjects were included (mean age 39 years, range $=29-46$ ). Restoration of active thumb flexion in patients was achieved by surgical transfer of one of the functioning elbow flexors (brachioradialis), to the paralyzed thumb flexor (flexor pollicis longus). We studied fMRI responses to isometric right-sided elbow flexion and key pinch, and examined the cortical representations within the left hemisphere somatomotor cortex a minimum of one year after surgery.

Results: Cortical activations elicited by elbow flexion did not differ in topography between patients and control participants. However, in contrast to control participants, patients' cortical thumb flexion activations were not topographically distinct from their elbow flexion activations.

Conclusion: This result speaks against a topographic reorganization in which the thumb region regains thumb control following surgical tendon transfer. Instead, our findings suggest a neuroplastic mechanism in which motor cortex resources previously dedicated to elbow flexion adapt to control the thumb.
\end{abstract}

Keywords: SCI, tendon transfer, plasticity, cortical reorganization, fMRI

\footnotetext{
${ }^{*}$ Corresponding author: Lina Bunketorp Käll, Assoc prof., Centre for Advanced Reconstruction of Extremities (C.A.R.E.), Sahlgrenska University Hospital/Mölndal, House U1, 6th floor,
}

43180 Mölndal, Sweden. Tel.: +46 709 723101; Fax: +46 31 873009; E-mail: lina.bunketorp-kall@neuro.gu.se. 


\section{Abbreviation}

\section{$\mathrm{CoG}$ center of gravity}

\section{Introduction}

Patients with a cervical spinal cord injury suffer severe upper limb function loss resulting in tetraplegia. In tetraplegia, there is injury to one of the eight cervical segments of the spinal cord and the lack of motor control and strength varies, depending on the level and severity of injury. Spinal cord lesions at the C6 or $\mathrm{C} 7$ level result in paralysis of the finger flexors, the finger extensors, the intrinsic muscles of the hand and partial or total triceps paralysis. The vast majority of spontaneous recovery (77\%) takes place within three months after the injury (Fawcett et al., 2007). Despite intensive rehabilitation therapy with the aim to enhance functional ability and independence, permanent upper limb function loss severely impairs the quality of life for affected individuals (Bryden et al., 2012). Consequently, restoring hand function is considered to be the highest priority among patients with tetraplegia, being prioritised above bowel, bladder, sexual function, or walking ability (Fridén \& Gohritz, 2015; Lo et al., 2016). Due to minimal loss of strength in elbow flexors and wrist extensors after spinal cord lesions at the $\mathrm{C} 6$ or $\mathrm{C} 7$ level, several highly successful surgical interventions have evolved. Surgical reconstruction of grip function in tetraplegia has constantly been developed since its introduction in the early 1970s. The basis for surgical restoration of the tetraplegic hand lies in mobilizing paralyzed joints where tendon transfers are the core traditional procedures (Bryden et al., 2012; Dunn et al., 2016; Fridén \& Gohritz, 2015; Skirven et al., 2011). Here, the distal end of a functionally intact muscle and its tendon are detached from its normal insertion, rerouted and reattached to the distal tendon of a paralyzed muscle to replace its original function (Skirven et al., 2011). Reconstruction of thumb flexion to create key pinch is preferably achieved by transferring one of the three elbow flexor muscles, the tendon of Brachioradialis (BR) to the tendon of the paralyzed thumb flexor; Flexor Pollicis Longus (FPL). Innovative single-stage combined procedures have developed from basic scientific research and clinical studies, and have been proven to offer considerable advantages over traditional approaches (Fridén \& Gohritz, 2015). Specifically, grip reconstruction is achieved through a combination of surgical procedures including the BR-FPL tendon transfer (Fridén et al., 2011).

Following surgery, patients are subjected to an intense rehabilitation program (Wangdell et al., 2016). Three weeks after surgery the patient is encouraged to use their restored grip functions in daily activities (Wangdell et al., 2016). Previous studies show that the restoration of grip function in tetraplegia leads to recognized functional benefits in activities of daily living and enhanced quality of life in individuals with tetraplegia (Bunketorp-Käll et al., 2017; Wangdell et al., 2013).

Based upon neuroimaging studies, the central nervous system (CNS) is now considered to be functionally dynamic. The CNS is adaptive, or plastic, and has some capacity to adapt or re-organize itself to compensate for the loss of function and/or maximize remaining functions in the event of an injury to the central nervous system. More specifically, the functional topography of the motor cortex (commonly called the motor homunculus or motor map) can be modified by a variety of manipulations such as peripheral or central injury, electrical stimulation, and behavioral experience (Nudo et al., 2001). Behavioral experiences that typically induce long-term plasticity in motor maps appear to be limited to those that entail the development of new motor skills (Nudo et al., 2001).

The execution of motor tasks involves several brain areas, in particular the primary motor cortex (M1). Somatotopic organization of M1 illustrates the fundamental organizational principle of functional specificity in the brain. Somatotopic organization refers to the anatomic mapping of motor functions within the CNS. Advances of brain mapping and neuroimaging have enabled studies of neural plasticity in the human brain (Kokotilo et al., 2009). A large body of literature has examined neuroplasticity changes and motor cortex reorganization as a consequence of SCI (Freund, P. et al., 2013; Kokotilo et al., 2009; Moxon et al., 2014; Nardone et al., 2013). Several studies have examined cortical reorganization of motor and sensory systems following SCI, showing that the CNS is adaptive, or plastic, and has some capacity to re-organize itself. (Freund, P. et al., 2013). With the exception of possible expansions of limb-specific activations (Curt et al., 2002; Jurkiewicz et al., 2007), the topography of the primary sensorimotor regions seems to remain largely intact (Corbetta et al., 2002; Shoham et al., 2001; Turner et al., 2003). However, due to varying protocols and conflicting results it is not possible to draw 
definite conclusions regarding the effects of SCI on brain reorganization (Kokotilo et al., 2009).

The human hand represents one of the most complex biological motor systems (Bezuhly et al., 2007). The way in which individuals with tetraplegia gain voluntary control over paralyzed functions following surgical tendon transfer, is an area of intense interest. Reconstructive surgery amounts to a unique, focused stimulus for brain plasticity and therefore yields a rare opportunity to characterize cortical plasticity in relation to the process of regaining limb function. Although tendon transfer has been performed in patients since the 1970s (Dunn et al., 2016), no previous studies have sought to delineate neuroplasticity in the restoration of upper limb function following tendon transfers in tetraplegia.

Given the preserved topography (Kikkert et al., 2016) and retained functionality of neuronal networks in spinal cord injured patients (HotzBoendermaker et al., 2008), it seems likely that regained limb control may be mediated by activation of that limb's specific cortical sensorimotor region. Supporting this view, acute stage cortical sensorimotor fMRI activation reductions due to SCI progressively enlarge during recovery, and ultimately normalize when motor ability is regained (Jurkiewicz et al., 2007). In this study, we used functional Magnetic Resonance Imaging (fMRI) with the aim to examine whether restoration of voluntary grip control through tendon transfer in patients with tetraplegia follows the topographic pattern. Specifically, we examined the hypothesis that regained thumb control after surgical BR-FPL transfer activates the thumb region of the primary motor cortex.

\section{Materials and methods}

\subsection{Participants}

This neuroimaging study was approved by the Regional Ethics Committee in Gothenburg, Sweden (Ref number: 309-16) and was conducted in accordance with the Helsinki Declaration (World Medical, 2013). Six right-handed males with tetraplegia with a mean age of 40 years (range $=31-48$ ) who underwent right side upper-limb grip reconstructive surgery at the Centre for Advanced Reconstruction of Extremities (C.A.R.E.), Sahlgrenska University Hospital, Sweden were recruited for the study. Informed consent was obtained from all participants. Eligibility criteria were: 1) Complete or incomplete SCIs with an injury level C4-C7 with intact BR control; 2) No motor function below the wrist prior to surgery; 3) Surgical BR to FPL tendon transfer restoration of thumb flexion, at least one year prior to the study; 4) No history of other medical or neurological disorders; 5) No vision impairments requiring glasses, and 6) Individual factors that preclude entering the fMRI environment (e.g. metal implants which are not compatible with the MRI environment, pacemaker, claustrophobia). A healthy control group, matched on age and gender, fulfilling eligibility criteria 4-6 was also recruited.

\subsection{Postoperative training regimen}

To optimize recruitment of the restored function after surgery, the rehabilitation program includes early motor reeducation under the guidance of a physiotherapist. The training regimen has previously been described in detail (Wangdell et al., 2016). Voluntary activation of a transferred tendon is facilitated by instructing the patient to recruit the function of the donor muscle. For example when BR (elbow flexor) is transferred in order to replace the action of the FPL (thumb flexor), the patient should perform flexion of the elbow against resistance, with the forearm in a semi-pronated position, and the thumb passively extended. Positioning the elbow in approximately 60 degrees of flexion is facilitative, since it optimizes the motor output of BR in its new location. The cognitive effort patients usually have to put into the motor re-learning indicates the significant mental load involved. Voluntary activation of restored functions are repeatedly rehearsed. This means that the brain has probably adapted to the peripheral redirection of the BR when external resistive force is no longer necessary.

\subsection{Motor tasks and experimental design}

Participants were positioned supine on the bed of the MRI scanner with their head immobilized in a tight-fitting head coil in order to minimize motion artifacts. A wedge pillow was positioned under the legs of participants. The right arm was held such that the elbow was resting against the scanner wall, the wrist straight, and the elbow was immobilized in a plastic splint at about $60^{\circ}$ of flexion (Johanson et al., 2006). The palm was positioned towards the midline of the body, and a small cube was attached to the index finger by a small strip of Velcro tape, in order to keep the thumb stabilized and somewhat elevated in relation to the index finger (Fig. 1A). 

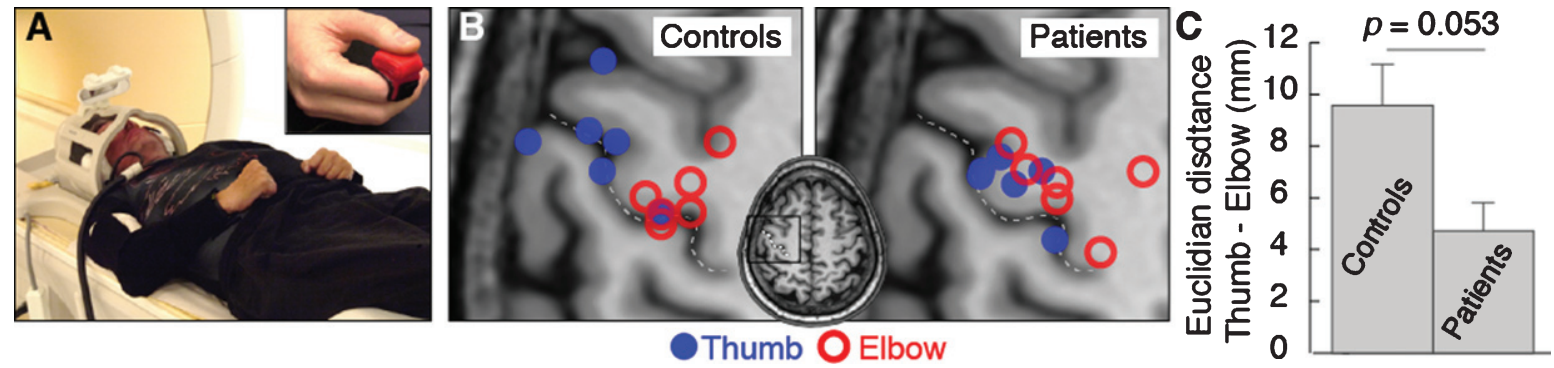

Fig. 1. (A) Experimental setup. (B) Activation center of gravity for thumb (blue) and elbow (red) flexion activations in control participants and patients. The central sulcus is indicated by the dotted line. (C) Mean cortical distance between thumb and elbow flexion activation centers of gravity for control participants and patients. Error bars indicate standard error of the mean. Significance of the planned comparison (one-tailed $t$-test) is shown.

Two isometric motor tasks were examined: thumb flexion and elbow flexion. Activation of a restored key pinch produces articulations mainly in the metacarpophalangeal joint due to balancing procedures including fusion of the carpometacarpal joint and a tenodesis in the interphalangeal joint (Fridén et al., 2011). Prior to the experiment, the maximal voluntary contraction during isometric activation of the key pinch was measured using a Pinch Gauge (North Coast Medical, Gilroy, USA) (Vanden Berghe et al., 1991). All subjects then trained to exert target forces corresponding to approximately $20 \%$ of the MVC for both motor tasks. Each scanning session in the fMRI protocol consisted of six runs, each including 10 pseudo-randomized 10 s blocks, with equal numbers of each movement. Each of the two motor tasks lasted 1 second, a speed that was found to be easy and comfortable to perform in pilot trials. The duration of each run was approximately 250 seconds. Across runs, each motor task was repeated 300 times. Participants were guided by auditory and visual cues presented on a screen viewed through a head-coil mounted mirror. A blinking graphic illustration was presented depicting either a key pinch or a flexed elbow, paced by digitized sound effects. The blocks were separated by an inter stimulus interval (ISI) of $12-15 \mathrm{~s}$, during which subjects were instructed to visualize a fixation ring and to remain motionless. All participants performed an in-scanner practice session prior to data collection and were reminded, between runs to avoid any movements not associated with the required tasks.

\subsection{MRI acquisition}

MRI was performed on a Philips Gyroscan 3T Achieva to obtain structural T1-weighted scans (flip angle $9^{\circ}$, echo time $3.285 \mathrm{~ms}$, repetition time $7.200 \mathrm{~ms}, 160$ sagittal slices with scan resolution $1.0 \times 1.0 \times 1.0 \mathrm{~mm}^{3}$ ) and $\mathrm{T} 2 *$-weighted gradient echo-planar imaging functional data ( 84 volumes, flip angle $70^{\circ}$, echo time $14 \mathrm{~ms}$, repetition time $3000 \mathrm{~ms}$, 45 axial slices, slice thickness $3 \mathrm{~mm}$, slice spacing $3 \mathrm{~mm}$, in-plane resolution $2.5 \times 2.5 \mathrm{~mm}^{2}$ ).

\subsection{Measures of hand function}

Prior to the fMRI scanning session, pinch grip strength was measured with the Pinch Gauge (North Coast Medical, Gilroy, USA) (Vanden Berghe et al., 1991). We also administered the Swedish tetraplegia surgery satisfaction questionnaire (Bunketorp-Käll et al., 2017), and assessed handedness using the Edinburgh inventory (Oldfield, 1971).

\subsection{MRI preprocessing and analysis}

All analyses were conducted in native space. Structural scans were reconstructed and segmented into surface representations in FreeSurfer (http:// surfer.nmr.mgh.harvard.edu/), and the segmented precentral gyrus was obtained and used as a mask in subsequent analyses (Makin et al., 2015). Preprocessing of functional data including realignment, co-registration and smoothing were performed in SPM12 using default parameters (http://www.fil. ion.ucl.ac.uk/spm/software/spm12/), and volumes with motion artifacts were identified using the Artifact Detection Toolbox (http://www.nitrc.org/) with default settings. General linear model analyses were conducted in SPM12, including boxcar regressors convolved with the canonical hemodynamic response function, as well as motion parameters and outlier 
volumes as regressors of no interest. All results were assessed at individual level family-wise error (FWE) corrected $p<0.05$.

\subsection{Statistical comparisons and computations}

In order to assess the topographic organization of the motor cortex, we computed maximum activation center of gravity $(\mathrm{CoG})$ coordinates (Makin et al., 2015). When this fell in the ventral third of the precentral gyrus, i.e. far from the upper limb representation, the second maximum activation cluster was selected (one patient, three control participants). The CoGs were then converted to the Montreal Neurological Institute (MNI) coordinate system for group comparisons. First, we examined the topographical organization in control participants. We conducted one-tailed paired $t$-tests to determine whether the thumb $\mathrm{CoG}$ was medial and ventral to the elbow CoG, as previously shown (Meier et al., 2008). To assess the degree of elbow and thumb separation in an unbiased fashion, we applied the k-means clustering algorithm in Matlab (The Mathworks), USA; version R2013a) to assign each CoG into one of two clusters, and permutation testing with 10,000 iterations was used to determine whether the algorithm performed significantly better than chance.

Second, we conducted a series of analyses to determine whether patients' activation patterns were consistent with the hypothesis. As above, we tested whether the thumb CoG was medial and ventral to the elbow CoG. We also tested whether patients' elbow and thumb CoGs differed in location from the controls' corresponding CoGs. Again, we applied $\mathrm{k}$-means clustering to assess whether the elbow and thumb flexion activations were topographically separate.

Further, we computed the Euclidian distances between the thumb and elbow CoGs for all participants to test whether the thumb-to-elbow cortical distances were comparable in patients and controls, and if there was a correlation between thumb-toelbow cortical distances and the time elapsed since surgery in patients. Similarly, we tested whether the distance from each patient's elbow CoG to the average control elbow $\mathrm{CoG}$ was smaller than the distance to the average control thumb CoG (i.e. if a patient's elbow $\mathrm{CoG}$ was closer to the control elbow $\mathrm{CoG}$ than the control thumb $\mathrm{CoG}$ ). We performed a similar comparison for each patient's thumb $\mathrm{CoG}$, ie. to determine whether a patient's thumb CoG was closer to the average control thumb $\mathrm{CoG}$ or the average control elbow CoG.

\section{Results}

\subsection{Participant demographics}

Six male patients, all right-handed, with a mean age of 40 years (range $=31-48$ ) participated in the study. The mean time since right-sided grip reconstruction was 7 years (range $=1-10$ ). Demographics, clinical characteristics and surgical procedures included in the right-sided grip reconstruction among the tetraplegic individuals are presented in Table 1. Prior to the grip reconstruction, two patients underwent reconstruction of the right elbow extensor (triceps) by a posterior deltoid-to-triceps transfer. The control group consisted of six right-handed male participants (mean age 39 years, range $=29-46$ ).

\subsection{Grip strength and satisfaction with surgery in the tetraplegic patient group}

All patients had successfully learned how to isolate the restored key pinch. The mean key pinch strength of the right hand among patients was $2.2 \mathrm{~kg}$ $(\mathrm{SD}=0.8)$. The corresponding values for controls were $10.9 \mathrm{~kg}(\mathrm{SD}=2.3)$. Patients were highly satisfied with the outcome of surgery. The mean score in the domains "general satisfaction" and "activity" was $43.3(\mathrm{SD}=1.9)$ out of 45 and $23.5(\mathrm{SD}=1.9)$ out of 25 , respectively. In the domain "occupation/schooling" the mean score was $17.6(\mathrm{SD}=7.9)$ out of 25. The mean score in the question about functional improvement was $5.0(\mathrm{SD}=0.0)$ out of 5 .

\subsection{Motion during brain scanning}

Patients exhibited more average linear, but not rotational, head movements than control participants. However, the differences were not significant when adjusting for multiple comparisons (all $p>0.2$ ). Out of the total 504 acquired fMRI volumes, an average of 1.50 (range $=0-2$ ) volumes during elbow and 0.67 (range $=0-4$ ) during thumb flexion were discarded due to motion artifacts in patients, and 0 and 0.33 (range $=0-2$ ), respectively, in control participants. There was no difference in discarded volumes between elbow and thumb flexion in patients (paired $t$-test, $p=0.292$ ). 
Table 1

Demographics, clinical characteristics and surgical procedures included in the right-sided grip reconstruction among the tetraplegic individuals

\begin{tabular}{llllcccc}
\hline Patient & Age & $\begin{array}{l}\text { Time since } \\
\text { SCI/surgery } \\
\text { (years) }\end{array}$ & $\begin{array}{l}\text { Cause of } \\
\text { injury }\end{array}$ & $\begin{array}{c}\text { BR function } \\
(0-5)^{1}\end{array}$ & $\begin{array}{c}\text { International } \\
\text { Classification }{ }^{2}\end{array}$ & $\begin{array}{c}\text { Level of } \\
\text { injury }\end{array}$ & Surgical procedures \\
\hline 1 & 31 & $13 / 1$ & Diving & 5 & 4 & C7 & tf, ff, ir, fpl-epl, ecu, cmcI \\
2 & 41 & $12 / 10$ & Fall & 5 & 3 & C6 & tf, ff, ir, fpl-epl, cmcI \\
3 & 48 & $6 / 5$ & Work-related & 5 & 4 & C7 & tf, ff, ir, fpl-epl, ecu, cmcI \\
4 & 39 & $10 / 7$ & Sport & 5 & 2 & C6 & tf, fpl-epl, elk, cmcI \\
5 & 41 & $26 / 10$ & Traffic & 5 & 2 & C6 & tf, ff, fpl-epl, ir \\
6 & 43 & $9 / 7$ & Diving & 5 & 4 & C7 & tf, ff, ir, fpl-epl, cmcI, ecu \\
\hline
\end{tabular}

SCI = Spinal Cord Injury, 1) Classified according to the Medical Research Council (MRC) system; 2) Description of motor groups according to the International Classification for Surgery (ICSHT) of the Hand in Tetraplegia; BR = brachioradialis; $\mathrm{tf}=$ thumb flexion reconstruction; $\mathrm{ff}=$ finger flexion reconstruction; ir = intrinsic reconstruction; elk = Extensor pollicis longus-loop-knot; fpl-epl $=$ Split FPL - EPL tenodesis; ecu $=$ Extensor Carpi Ulnaris tenodesis; $\mathrm{cmcI}=$ arthrodesis of carpometacarpal $(\mathrm{CMC})$ joint $\mathrm{I}$.

\subsection{Brain activations}

Thumb and elbow movements elicited extensive precentral gyrus activations in all participants (FWE $p<0.05$ ). Consistent with the previously demonstrated topographic organization of the motor cortex (Meier et al., 2008), control participant thumb CoGs were significantly more distal (one-tailed paired $t$-test, $p=0.017$, Bonferroni corrected for three comparisons) and ventral (corrected $p=0.032$ ) than their elbow flexion CoGs. There was a trend towards a more anterior location (uncorrected $p=0.029$, corrected $p=0.086$ ) (Fig. 1B). The k-means clustering algorithm correctly assigned the elbow and thumb flexion CoGs to topographically separate clusters $(p=0.014)$.

In patients, the thumb flexion CoGs were not more distal (one-tailed paired $t$-test, $p=0.098$, Bonferroni corrected for three comparisons) or anterior (corrected $p=0.246$ ) than their elbow flexion $\mathrm{CoG}$, but the thumb CoG was more ventral (one-tailed paired $t$-test, corrected $p=0.020$ ) (Fig. 1B). Consistently, the k-means clustering algorithm failed to correctly assign the CoGs $(p=0.247)$.

For elbow flexion activations, patients' and control participants' CoGs did not differ in any dimension (all uncorrected $p>0.4$ ) (Fig. 1B). For thumb flexion activations, patient $\mathrm{CoG}$ were significantly more medial (one-tailed $t$-test $p=0.049$, corrected for three multiple comparisons) than control participants thumb CoGs, and there were trends towards a more posterior (one-tailed $t$-test, uncorrected $p=0.070$ ) and dorsal (one-tailed $t$-test, uncorrected $p=0.050$ ) location. That is, patient thumb CoG was located towards control participant elbow CoG (Fig. 1B).

The distance between the thumb and elbow flexion $\mathrm{CoG}$ was smaller in patients (mean $4.72 \mathrm{~mm}$, SD
3.80) than in control participants (mean $9.57 \mathrm{~mm}, \mathrm{SD}$ 5.53) (one-tailed $t$-test, $p=0.053$ ) (Fig. 1C). There was no correlation between thumb to elbow CoG distance and duration since surgery in patients $(r=0.248$, $p=0.636$ ).

Patients' elbow CoGs were located closer to the average control participants' elbow CoGs than the thumb CoGs: the Euclidian distance between patients' and average control participants' elbow CoGs was significantly smaller than the distance to the control participants' thumb CoGs (one-tailed paired $t$-test, $p=0.003$ ) (Fig. 2). However, patients' thumb CoGs were not located closer to the average control participant thumb CoG than their elbow CoG (one-tailed paired $t$-test, $p=0.695$ ) (Fig. 2).

\section{Discussion}

We examined primary motor cortex plasticity associated with re-established motor function after surgical tendon transfer in tetraplegia, and tested the hypothesis that regained thumb control is mediated by reactivations of the corresponding topographic area of the motor cortex. Our results do not support this hypothesis: patients' thumb activations were not topographically distinct from their elbow activations. In patients, the activation did not match the topographic organization of controls. Our findings thus suggest plasticity in which the cortical region for elbow flexion may have adapted to also control the paralyzed thumb muscle. The average number of years elapsed since surgery and the post-surgical rehabilitation did vary between 1-10 years, but we found no correlation between time elapsed since surgery and cortical alterations. 


\section{Cortical distance analyses}

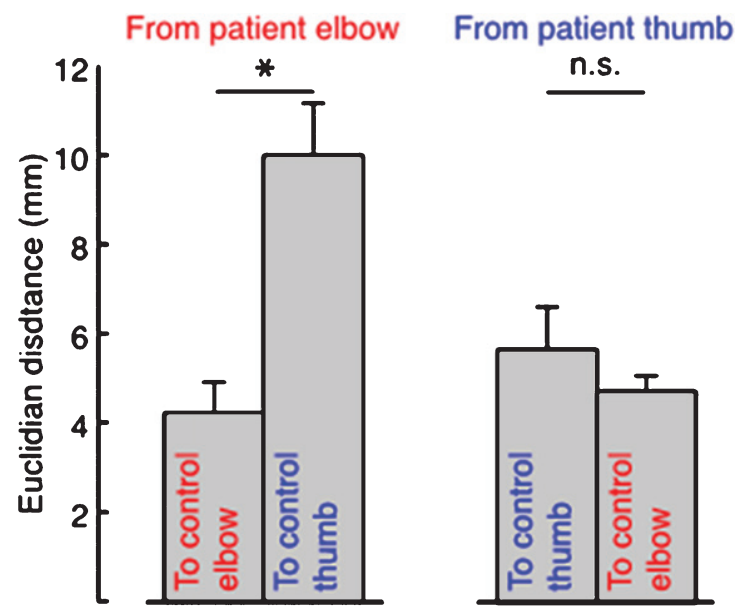

Fig. 2. Average cortical distances between patients' elbow and thumb flexion activation centers of gravity (CoGs) and control participant's CoGs, showing that the distance from patient's elbow $\mathrm{CoG}$ to the average control participant's elbow $\mathrm{CoG}$ was significantly smaller than the distance from patient's elbow CoG to the average control thumb CoG, whereas patient's thumb CoG was not more near neither the average control's thumb or elbow CoG. Error bars indicate standard error of the mean. Significance of planned comparisons (one-tailed $t$-tests) are denoted as follows: ${ }^{*} p<0.05$, n.s. $p>0.05$.

Patient cortical thumb flexion representation was significantly more patchy, seemed similar to the elbow representation, and located more lateral and ventral than in controls. This suggests a functional remapping of elbow neurons corresponding to the transferred BR muscle area. The BR exerts a flexion moment at the level of the elbow (Johanson et al., 2011). A well-controlled recruitment for a key pinch restoration requires a synergistic co-contraction of either the triceps or transferred posterior deltoid. This is necessary to prevent excessive elbow flexion generated by the BR (Johanson et al., 2006; Johanson et al., 2011; Waters et al., 1990). In healthy subjects, the BR does not contract during thumb flexion (Waters et al., 1990). We found no difference in elbow flexion between patients and control participants, despite the surgical alteration of BR in patients. Since the neurological level of injury was C6 and C7 among patients, the function of their elbow flexors was unaffected. Thus, the resisted elbow flexion task recruits all elbow flexors in the arm in a similar way in both patients and controls.

To facilitate motor re-education and voluntary recruitment of transferred tendons, early active postoperative training is required (Fridén \& Gohritz,
2015; Wangdell et al., 2016). Early mobilization also helps in preventing adhesions. Starting the day after surgery, patients perform intermittent exercises. The aim is to isolate recruitment of the transferred donor muscle in its new location and coordinate movements with new synergists during ADL. This motor reeducation sheds light on the brain's capacity to adapt to peripheral changes. Moreover, previous studies demonstrate that immobilization of the upper extremity in healthy subjects causes deprivation of the sensorimotor network by specific neuroanatomical changes in the cortical regions responsible for the sensorimotor control of the upper limb (Langer et al., 2012). This further supports the importance of early active training after tendon transfer (Wangdell et al., 2016).

It is noteworthy to mention that the functional organization of M1 is much more complex than has previously been described (Plow et al., 2010; Schieber, 2001). More recently, muscle representations are shown to overlap; individual muscles have multiple representations that intermingle with representations of related movements or parts forming a complex mosaic pattern (Graziano \& Aflalo, 2007; Hosp \& Luft, 2011). There are individual corticospinal neurons that diverge to multiple motor neuron pools and horizontal fibers that interconnect distributed representations. This complex organization may underlie functional plasticity in local sub-regions of the motor cortex (Nudo et al., 2001). There are studies on complete spinal cord injuries that show activation in the motor network several years after a spinal injury when they mentally imagine themselves moving a paralyzed body part (Sabbah et al., 2002). Thus, the mental representation of the movement remains, despite the complete spinal cord injury. However, it has been shown that activation of the sensory and motor cortex is considerably weaker if one only imagines the movement, and activity in the cerebellum is missing (Nyberg et al., 2001). In addition, a previous neurophysiological study in individuals with SCI demonstrated long-lasting involuntary activity at rest in muscles innervated from close to, within or below the level of the injury (Zoghi et al., 2016). This indicates a high state of central excitability and may reflect a loss of discrete activation of the transferred BR muscle in its new role of controlling two different movements.

There are a number of limitations to this study. As a result of the rarity of tetraplegia combined with the strict inclusion criteria, only six patients were examined. Taking into account the number of people who sustain a SCI worldwide, restorative surgery 
is an underutilized procedure, although widely advocated (Fridén \& Gohritz, 2015). The reason for this is suggested to be skepticism among patients, therapists, and rehabilitation physicians due to inadequate information as well as inadequate referral networks (Curtin et al., 2005). Nevertheless, the resulting homogenous patient group and highly focused hypothesis allowed a within-subject analysis with comparatively robust results, suggesting that motor cortex plasticity is largely consistent across patients. Conversely, the study design did not allow exploration of brain regions or circuits outside the motor cortex, and additional studies in larger samples are required to examine brain-wide mechanisms of plasticity following tendon transfer.

In addition, we observed that patients moved more than the control group. However, rigorous control for movement was included in the analysis of results so selective effects of motion are unlikely. Despite these limitations, this study suggests that the gain of limb function following surgical tendon transfer and early active training is mediated through adaptive changes in the central nervous system that do not follow pre-existing topographic maps. This finding reflects adaptive changes in the central nervous system as a response to extrinsic stimuli (Chen et al., 2003; Cramer et al., 2011), and has a number of clinical implications. Cortical adaptation is a critical facilitator of successful motor relearning, and delineation of the underlying mechanisms may lead to improved rehabilitation approaches and biomarkers (Nardone et al., 2013). Our findings also suggest that successful tendon transfer restoration of voluntary limb control is possible despite any atrophy of cortical regions associated with the unused limb (Freund, Patrick et al., 2013; Freund et al., 2011; Haefeli et al., 2016; Wrigley et al., 2009). Surgical reconstruction of grip functions remains possible, even decades after cervical spinal cord paralysis (Fridén \& Gohritz, 2015). All persons living with impaired upper limb functions resulting from SCI should therefore be assessed and informed of possible reconstructive options (Bunketorp-Käll et al., 2017; Fridén \& Gohritz, 2015).

Finally, our results are particularly relevant to neuroprosthetics research (Davis et al., 2016). The finding that the motor cortex may adapt to allow bodypart specific regions to gain control over other limbs suggests that cortical implants in one region may effectively control multiple types of robotic movements. Indeed, such cortical adaptation may underlie the ability of tetraplegic patients with implants in the hand area of the motor cortex only to perform sophisticated reaching movements with robotic arms (Hochberg et al., 2012).

\section{Conclusion}

Findings from the current study suggests that restored thumb flexion following surgical transfer of an elbow flexor is mediated through adaptive changes in the central nervous system that do not follow pre-existing topographic maps. Our results suggest a neuroplastic mechanism in which cortical areas previously dedicated to elbow flexion may have adapted to control thumb flexion.

\section{Acknowledgments}

This work was supported by funding from Promobilia foundation, Norrbacka-Eugenia foundation, Neuroförbundet (NEURO, Sweden), Capio Research Foundation and The Swedish Association for Survivors of Accident and Injury.

\section{References}

Bezuhly, M., Sparkes, G.L., Higgins, A., Neumeister, M.W., \& Lalonde, D.H. (2007). Immediate thumb extension following extensor indicis proprius-to-extensor pollicis longus tendon transfer using the wide-awake approach. Plastic and Reconstructive Surgery, 119(5), 1507-1512.

Bryden, A.M., Peljovich, A.E., Hoyen, H.A., Nemunaitis, G., Kilgore, K.L., \& Keith, M.W. (2012). Surgical restoration of arm and hand function in people with tetraplegia. Topics in Spinal Cord Injury Rehabilitation, 18(1), 43-49.

Bunketorp-Käll, L., Wangdell, J., Reinholdt, C., \& Fridén, J. (2017). Satisfaction with upper limb reconstructive surgery in individuals with tetraplegia: The development and reliability of a Swedish self-reported satisfaction questionnaire. Spinal Cord, 55(7), 664-671.

Chen, R., Anastakis, D.J., Haywood, C.T., Mikulis, D.J., \& Manktelow, R.T. (2003). Plasticity of the human motor system following muscle reconstruction: A magnetic stimulation and functional magnetic resonance imaging study. Clinical $\mathrm{Neu}$ rophysiology, 114(12), 2434-2446.

Corbetta, M., Burton, H., Sinclair, R.J., Conturo, T.E., Akbudak, E., \& McDonald, J.W. (2002). Functional reorganization and stability of somatosensory-motor cortical topography in a tetraplegic subject with late recovery. Proceedings of the National Academy of Sciences, 99(26), 17066-17071.

Cramer, S.C., Sur, M., Dobkin, B.H., O’brien, C., Sanger, T.D., Trojanowski, J.Q., Rumsey, J.M., Hicks, R., Cameron, J., \& Chen, D. (2011). Harnessing neuroplasticity for clinical applications. Brain, 134(6), 1591-1609. 
Curt, A., Alkadhi, H., Crelier, G.R., Boendermaker, S.H., Hepp-Reymond, M.C., \& Kollias, S.S. (2002). Changes of non-affected upper limb cortical representation in paraplegic patients as assessed by fMRI. Brain, 125(11), 2567-2578.

Curtin, C.M., Gater, D.R., \& Chung, K.C. (2005). Upper extremity reconstruction in the tetraplegic population, a national epidemiologic study. Journal of Hand Surgery, 30(1), 94-99.

Davis, T., Wark, H., Hutchinson, D., Warren, D., O’Neill, K., Scheinblum, T., Clark, G.A., Normann, R., \& Greger, B. (2016). Restoring motor control and sensory feedback in people with upper extremity amputations using arrays of 96 microelectrodes implanted in the median and ulnar nerves. Journal of neural engineering, 13(3), 036001.

Dunn, J.A., Sinnott, K.A., Rothwell, A.G., Mohammed, K.D., \& Simcock, J.W. (2016). Tendon transfer surgery for people with tetraplegia: An overview. Archives of Physical Medicine and Rehabilitation, 97(6), S75-S80.

Fawcett, J.W., Curt, A., Steeves, J.D., Coleman, W.P., Tuszynski, M.H., Lammertse, D., Bartlett, P.F., Blight, A.R., Dietz, V., Ditunno, J., Dobkin, B.H., Havton, L.A., Ellaway, P.H., Fehlings, M.G., Privat, A., Grossman, R., Guest, J.D., Kleitman, N., Nakamura, M., Gaviria, M., \& Short, D. (2007). Guidelines for the conduct of clinical trials for spinal cord injury as developed by the ICCP panel: Spontaneous recovery after spinal cord injury and statistical power needed for therapeutic clinical trials. Spinal Cord, 45(3), 190-205.

Freund, P., Curt, A., Friston, K., \& Thompson, A. (2013). Tracking changes following spinal cord injury: Insights from neuroimaging. Neuroscientist, 19(2), 116-128.

Freund, P., Weiskopf, N., Ashburner, J., Wolf, K., Sutter, R., Altmann, D.R., Friston, K., Thompson, A., \& Curt, A. (2013). MRI investigation of the sensorimotor cortex and the corticospinal tract after acute spinal cord injury: A prospective longitudinal study. The Lancet Neurology, 12(9), 873-881.

Freund, P., Weiskopf, N., Ward, N.S., Hutton, C., Gall, A., Ciccarelli, O., Craggs, M., Friston, K., \& Thompson, A.J. (2011). Disability, atrophy and cortical reorganization following spinal cord injury. Brain, 134, 1610-1622.

Fridén, J., \& Gohritz, A. (2015). Tetraplegia Management Update. Journal of Hand Surgery, 40(12), 2489-2500.

Fridén, J., Reinholdt, C., Turcsanyii, I., \& Gohritz, A. (2011). A single-stage operation for reconstruction of hand flexion, extension, and intrinsic function in tetraplegia: The alphabet procedure. Techniques in Hand and Upper Extremity Surgery, 15(4), 230-235.

Graziano, M.S., \& Aflalo, T.N. (2007). Mapping behavioral repertoire onto the cortex. Neuron, 56(2), 239-251.

Haefeli, J., Huie, J.R., Morioka, K., \& Ferguson, A.R. (2016). Assessments of sensory plasticity after spinal cord injury across species. Neuroscience Letters, 652 (23), 74-81.

Hochberg, L.R., Bacher, D., Jarosiewicz, B., Masse, N.Y., Simeral, J.D., Vogel, J., Haddadin, S., Liu, J., Cash, S.S., \& van der Smagt, P. (2012). Reach and grasp by people with tetraplegia using a neurally controlled robotic arm. Nature, 485(7398), 372-375.

Hosp, J.A., \& Luft, A.R. (2011). Cortical plasticity during motor learning and recovery after ischemic stroke. Neural Plasticity, 2011,1-9.

Hotz-Boendermaker, S., Funk, M., Summers, P., Brugger, P., Hepp-Reymond, M.C., Curt, A., \& Kollias, S.S. (2008).
Preservation of motor programs in paraplegics as demonstrated by attempted and imagined foot movements. Neuroimage, 39(1), 383-394.

Johanson, M.E., Hentz, V.R., Smaby, N., \& Murray, W.M. (2006). Activation of brachioradialis muscles transferred to restore lateral pinch in tetraplegia. Journal of Hand Surgery, 31(5), 747-753.

Johanson, M.E., Murray, W.M., \& Hentz, V.R. (2011). Comparison of wrist and elbow stabilization following pinch reconstruction in tetraplegia. Journal of Hand Surgery, 36(3), 480-485.

Jurkiewicz, M.T., Mikulis, D.J., McIlroy, W.E., Fehlings, M.G., \& Verrier, M.C. (2007). Sensorimotor cortical plasticity during recovery following spinal cord injury: A longitudinal fMRI study. Neurorehabilitation and Neural Repair, 21(6), 527-538.

Kikkert, S., Kolasinski, J., Jbabdi, S., Tracey, I., Beckmann, C.F., Johansen-Berg, H., \& Makin, T.R. (2016). Revealing the neural fingerprints of a missing hand. Elife, 5, e15292.

Kokotilo, K.J., Eng, J.J., \& Curt, A. (2009). Reorganization and preservation of motor control of the brain in spinal cord injury: A systematic review. Journal of Neurotrauma, 26(11), 21132126.

Langer, N., Hanggi, J., Muller, N.A., Simmen, H.P., \& Jancke, L. (2012). Effects of limb immobilization on brain plasticity. Neurology, 78(3), 182-188.

Lo, C., Tran, Y., Anderson, K., Craig, A., \& Middleton, J. (2016). Functional priorities in persons with spinal cord injury: Using discrete choice experiments to determine preferences. Journal of Neurotrauma, 1;33(21), 1958-1968.

Makin, T.R., Scholz, J., Slater, D.H., Johansen-Berg, H., \& Tracey, I. (2015). Reassessing cortical reorganization in the primary sensorimotor cortex following arm amputation. Brain, 138(8), 2140-2146.

Moxon, K.A., Oliviero, A., Aguilar, J., \& Foffani, G. (2014). Cortical reorganization after spinal cord injury: Always for good? Neuroscience, 283, 78-94.

Nardone, R., Höller, Y., Brigo, F., Seidl, M., Christova, M., Bergmann, J., Golaszewski, S., \& Trinka, E. (2013). Functional brain reorganization after spinal cord injury: Systematic review of animal and human studies. Brain Research, 1504, 58-73.

Nudo, R.J., Plautz, E.J., \& Frost, S.B. (2001). Role of adaptive plasticity in recovery of function after damage to motor cortex. Muscle and Nerve, 24(8), 1000-1019.

Nyberg, L., Petersson, K.M., Nilsson, L.-G., Sandblom, J., Åberg, C., \& Ingvar, M. (2001). Reactivation of motor brain areas during explicit memory for actions. Neuroimage, 14(2), 521528.

Oldfield, R.C. (1971). The assessment and analysis of handedness: The Edinburgh inventory. Neuropsychologia, 9(1), 97-113.

Plow, E.B., Arora, P., Pline, M.A., Binenstock, M.T., \& Carey, J.R. (2010). Within-limb somatotopy in primary motor cortex-revealed using fMRI. Cortex, 46(3), 310-321.

Sabbah, P., De Schonen, S., Leveque, C., Gay, S., Pfefer, F., Nioche, C., Sarrazin, J.-L., Barouti, H., Tadie, M., \& Cordoliani, Y.-S. (2002). Sensorimotor cortical activity in patients with complete spinal cord injury: A functional magnetic resonance imaging study. Journal of Neurotrauma, 19(1), 53-60. 
Schieber, M.H. (2001). Constraints on somatotopic organization in the primary motor cortex. Journal of Neurophysiology, 86(5), 2125-2143.

Shoham, S., Halgren, E., Maynard, E.M., \& Normann, R.A. (2001). Motor-cortical activity in tetraplegics. Nature, 413(6858), 793

Skirven, T.M., Osterman, A.L., Fedorczyk, J., \& Amadio, P.C. (2011). Rehabilitation of the hand and upper extremity, 2volume set: Expert consult: Elsevier Health Sciences.

Turner, J.A., Lee, J.S., Schandler, S.L., \& Cohen, M.J. (2003). An fMRI investigation of hand representation in paraplegic humans. Neurorehabilitation and Neural Repair, 17(1), 3747.

Vanden Berghe, A., Van Laere, M., Hellings, S., \& Vercauteren, M. (1991). Reconstruction of the upper extremity in tetraplegia: Functional assessment, surgical procedures and rehabilitation. Paraplegia, 29(2), 103-112.

Wangdell, J., Bunketorp-Käll, L., Koch-Borner, S., \& Fridén, J. (2016). Early active rehabilitation after grip reconstructive surgery in tetraplegia. Archives of Physical Medicine and Rehabilitation, 97(6), S117-S125.
Wangdell, J., Carlsson, G., \& Fridén, J. (2013). Enhanced independence: Experiences after regaining grip function in people with tetraplegia. Disability and Rehabilitation, 35(23), 19681974.

Waters, R.L., Stark, L.Z., Gubernick, I., Bellman, H., \& Barnes, G. (1990). Electromyographic analysis of brachioradialis to flexor pollicis longus tendon transfer in quadriplegia. Journal of Hand Surgery, 15(2), 335-339.

World Medical, A. (2013). World Medical Association Declaration of Helsinki: Ethical principles for medical research involving human subjects. JAMA, 310(20), 2191-2194.

Wrigley, P.J., Gustin, S.M., Macey, P.M., Nash, P.G., Gandevia, S.C., Macefield, V.G., Siddall, P., \& Henderson, L.A. (2009). Anatomical changes in human motor cortex and motor pathways following complete thoracic spinal cord injury. Cerebral Cortex, 19(1), 224-232.

Zoghi, M., Galea, M., \& Morgan, D. (2016). Brain motor control assessment of upper limb function in patients with spinal cord injury. Journal of Spinal Cord Medicine, 39(2), 162-174. 